\title{
Study to Evaluate the Histopathological Spectrum of Hepatic Lesions in Liver Biopsies in a Tertiary Care Hospital
}

\author{
Neekita Shriram Agrawal, M. Banyameen Iqbal*, Amardeep Ajinath Patil, Kunjal Mukesh Karia and Harsh Kumar \\ Department of Pathology, Dr. D.Y.Patil Medical College, Hospital and Research Centre, Pimpri, Pune-18,India
}

\section{ABSTRACT}

Background: Liver is affected by a wide spectrum of various primary and secondary diseases. These diseases can be diagnosed with the help of either clinical, biochemical or radiological examinations. But, liver biopsy remains the mainstay in diagnosis as well as management of various liver diseases.

Methods: A prospective study which included 65 liver biopsies. The sections were examined and the histopathological findings were recorded

Result: Out of 65 liver biopsies, $4.6 \%$ were inadequate for histopathological study. The various histopathological findings included secondary tumour deposits $(40.0 \%)$, primary hepatic tumours $(12.3 \%)$, hepatitis $(16.9 \%)$, cirrhosis $(12.3 \%)$, extrahepatic biliary atresia $(6.15 \%)$, secondary biliary cirrhosis $(3.0 \%)$, glycogen storage disease $(1.5 \%)$, cystic hydatid disease (1.5\%) and fatty liver (1.5\%).

Conclusion: The histopathological study of liver biopsy forms the cornerstone in the diagnosis and management of various hepatic diseases.

Keywords: Hepatic Lesions, Histopathological Spectrum, Liver Biopsy

\section{Introduction}

Liver is a primary organ for various metabolic activities of the body. It is exposed to various metabolic, toxic, infectious and neoplastic insults. ${ }^{[1]}$ Thus, a spectrum of various primary as well as secondary diseases can affect liver. The common primary liver diseases are hepatitis, nonalcoholic fatty liver disease (NAFLD), alcoholic liver disease (ALD) and hepatocellular carcinoma (HCC). Secondary hepatic involvement can be due to alcoholism, extrahepatic infections or metastatic spread of various primary malignancies. ${ }^{[2]}$

Liver biopsy is an essential tool in the diagnosis and management of liver diseases as liver function tests (LFT) alone are not diagnostically specific. ${ }^{[3]}$ Any significantly abnormal LFT and/or radiological finding with clinical findings of anaemia, jaundice, hepatomegaly, ascites and splenomegaly warrants a liver biopsy. The ease as well as it being a relatively safe procedure has made it a method of choice for diagnosis and subsequent management of hepatic lesions. Thus, histopathological study of liver biopsy is very common and is frequently done for diagnosis of different hepatic lesions.

\section{Materials and Methods}

The study was conducted on 65 liver biopsies prospectively in the department of pathology at a tertiary care centre over a period of one year (May 2016 to May 2017). The clinical and radiological findings with LFT results were noted in all the cases. Formalin fixed liver biopsy tissues were processed routinely. These tissues were dehydrated with ascending grades of alcohol, cleared with xylene, and embedded in paraffin to prepare blocks. The blocks were then cut into sections of 2-5 micrometre thickness using a microtome. These sections were stained with Haematoxylin and Eosin (H\&E) and were examined under the microscope. Some special stains like Reticulin, Periodic Acid Schiff (PAS), Masson Trichrome (MT), Prussian blue (Perl's) were used in selected cases. The findings were recorded and analyzed.

\section{Result}

In this study, age ranged from 3 months to 80 years. Mean age was 42.5 years. Majority of patients belonged to the $5^{\text {th }}$ and $6^{\text {th }}$ decade of life. Out of 65 cases, $43.07 \%$ were males and $56.92 \%$ were females. The male to female ratio was 0.7. [Table 1, Figure 1]

Hepatic tumours were the commonest finding 52.30\%, of which, $76.47 \%$ were secondaries and $23.52 \%$ were primary hepatic tumours. This was followed by hepatitis which included viral hepatitis, alcoholic hepatitis and neonatal hepatitis. Various other lesions diagnosed were cirrhosis, extrahepatic biliary atresia, secondary biliary cirrhosis, fatty liver, glycogen storage disease and cystic hydatid disease of liver. [Table 2, Figure 2] Our study revealed hepatic lesions of neoplastic etiology are at an increasing verge as compared to infective or metabolic causes. Of the neoplastic insults, secondary hepatic involvement by metastatic spread of various primary malignancies has become more common than primary hepatic neoplasms. 
Table 1: Age and Sex wise Distribution of Various Hepatic Lesions.

\begin{tabular}{|l|c|c|c|c|}
\hline Age Group & Male & Female & No. of Cases & $\begin{array}{c}\text { Percentage of Total } \\
\text { Cases }\end{array}$ \\
\hline $0-10$ & 04 & 04 & 08 & 12.30 \\
\hline $11-20$ & 00 & 03 & 03 & 04.61 \\
\hline $21-30$ & 02 & 06 & 08 & 12.30 \\
\hline $31-40$ & 04 & 04 & 08 & 12.30 \\
\hline $41-50$ & 07 & 04 & 11 & 16.92 \\
\hline $51-60$ & 05 & 06 & 11 & 16.92 \\
\hline $61-70$ & 04 & 09 & 13 & 20.00 \\
\hline $71-80$ & 02 & 01 & 03 & 04.61 \\
\hline Total No. of Cases & $\mathbf{2 8}(\mathbf{4 3 . 0 7 \% )}$ & $\mathbf{3 7}(\mathbf{5 6 . 9 2} \%)$ & $\mathbf{6 5}$ & $\mathbf{1 0 0 . 0}$ \\
\hline
\end{tabular}

Table 2: Frequency of Various Histopathological Diagnosis.

\begin{tabular}{|l|c|c|}
\hline Histopathological Diagnosis & No. of Cases & Percentage of Total Cases \\
\hline Hepatic Secondaries & 26 & 40.00 \\
\hline Primary Hepatic Tumour & 08 & 12.30 \\
\hline Cirrhosis & 08 & 03.30 \\
\hline Secondary Biliary Cirrhosis & 02 & 07.69 \\
\hline Viral Hepatitis & 05 & 06.15 \\
\hline Alcoholic Hepatitis & 04 & 03.07 \\
\hline Neonatal Hepatitis & 02 & 06.15 \\
\hline Extrahepatic Biliary Atresia & 04 & 01.53 \\
\hline Glycogen Storage Disease & 01 & 01.53 \\
\hline Fatty Liver & 01 & 01.53 \\
\hline Cystic Hydatid Disease & 01 & 04.61 \\
\hline Inadequate & 03 & 100.0 \\
\hline Total & 65 & \\
\hline
\end{tabular}

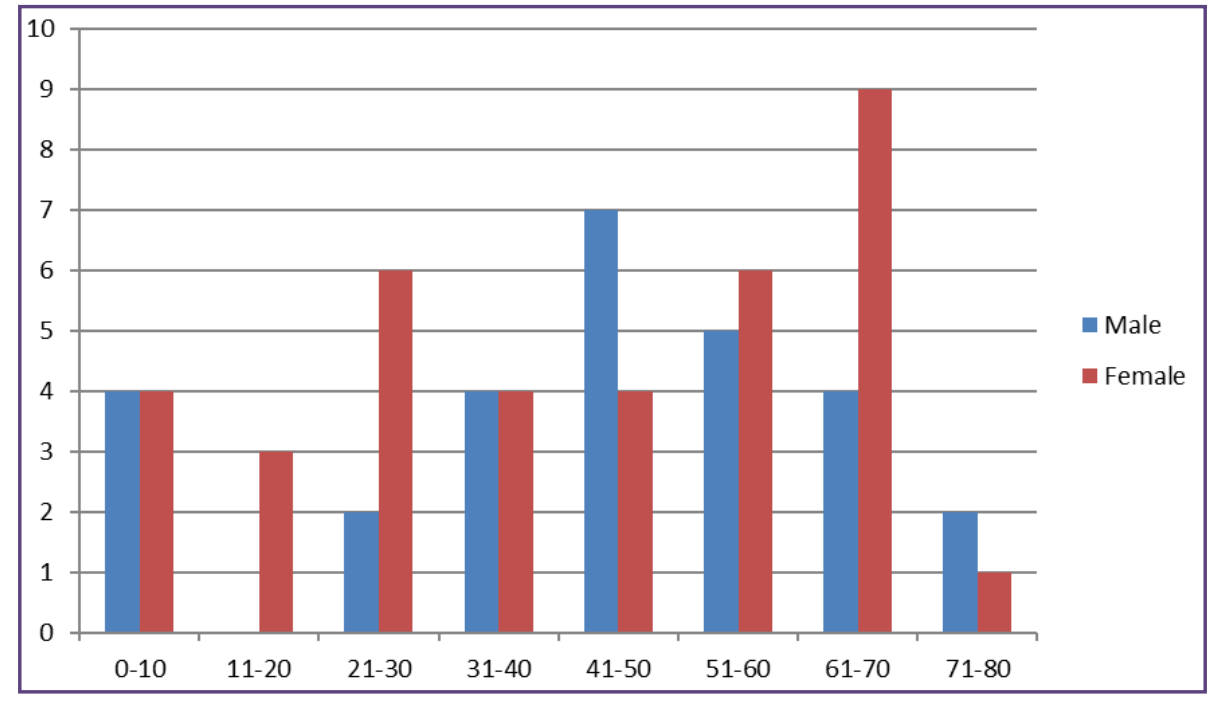

Fig. 1: Distribution of hepatic lesions in relation to age and sex. 


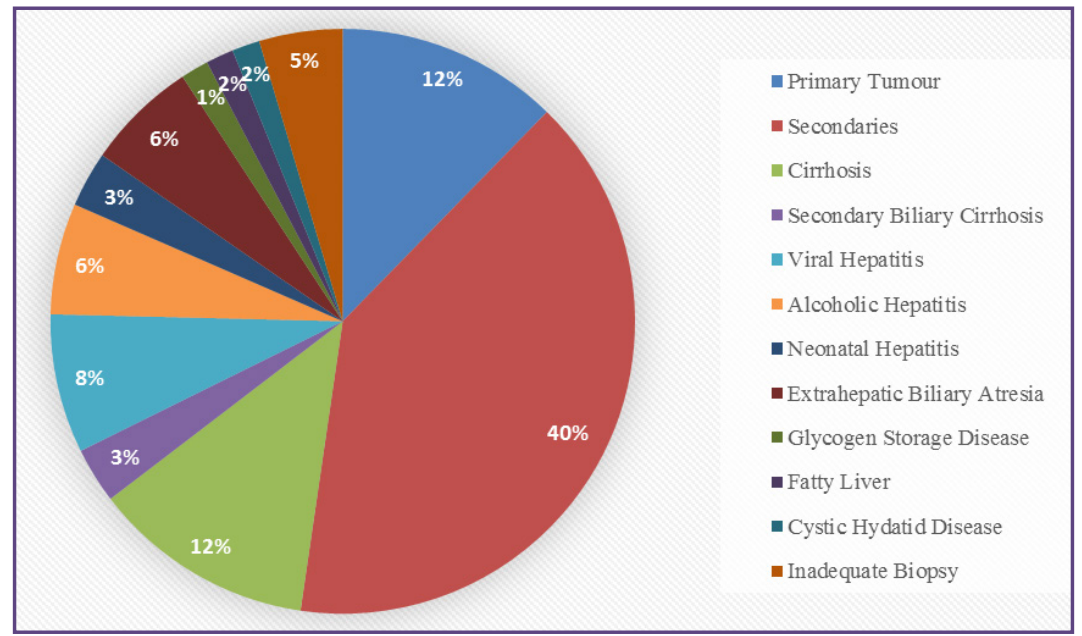

Fig. 2: Frequency of distribution of hepatic lesions.

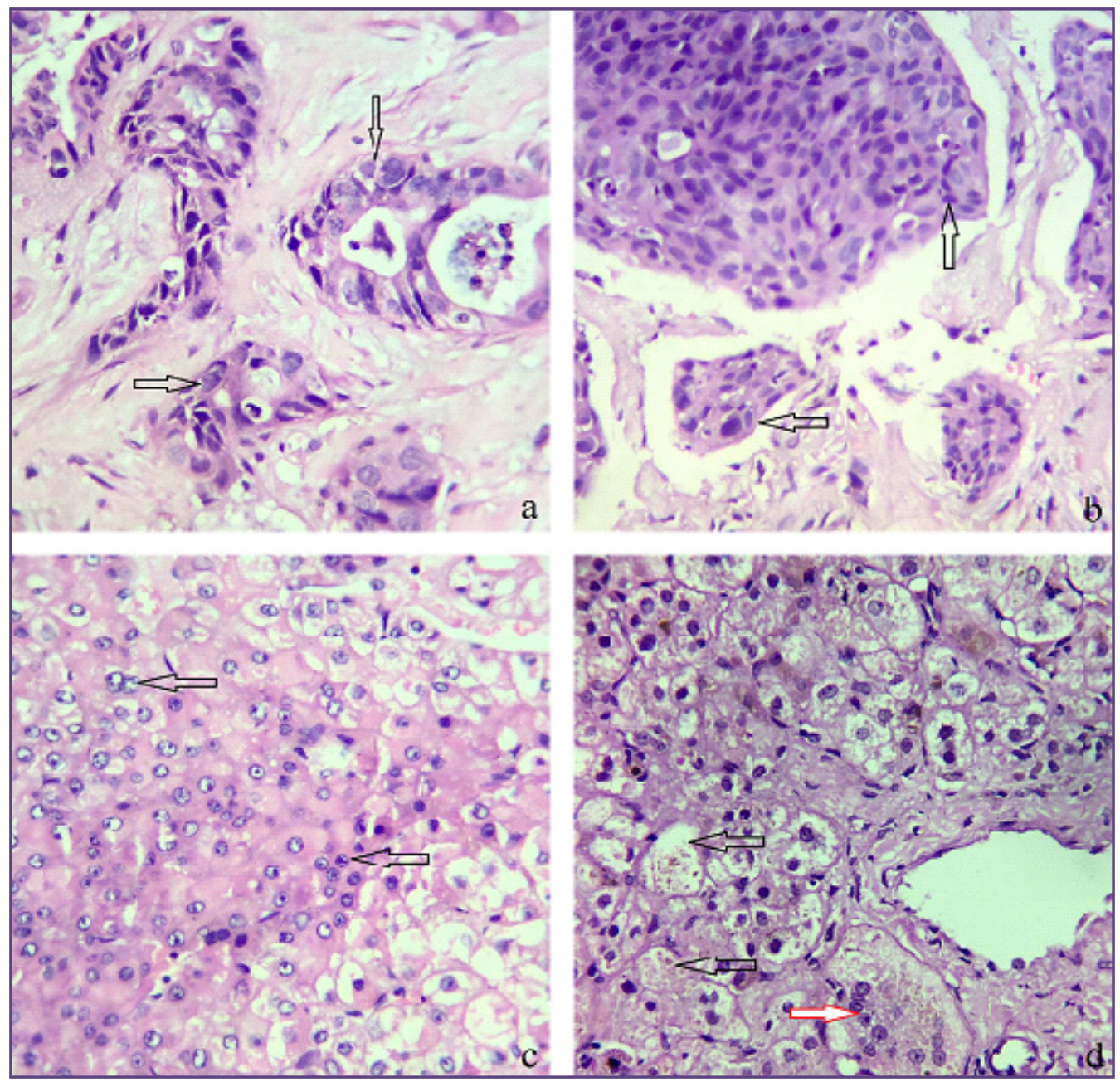

Fig. 3: Photomicrograph of metastatic adenocarcinoma deposits (arrows) in liver (a; H\&E, x40), metastatic deposits of squamous cell carcinoma (arrows) (b; H\&E, x40), hepatocellular carcinoma showing tumour cells (arrows) (c; H\&E, x40), neonatal hepatitis showing cholestasis (black arrows) and giant cell transformation (red arrow) (d; H\&E, x40). 


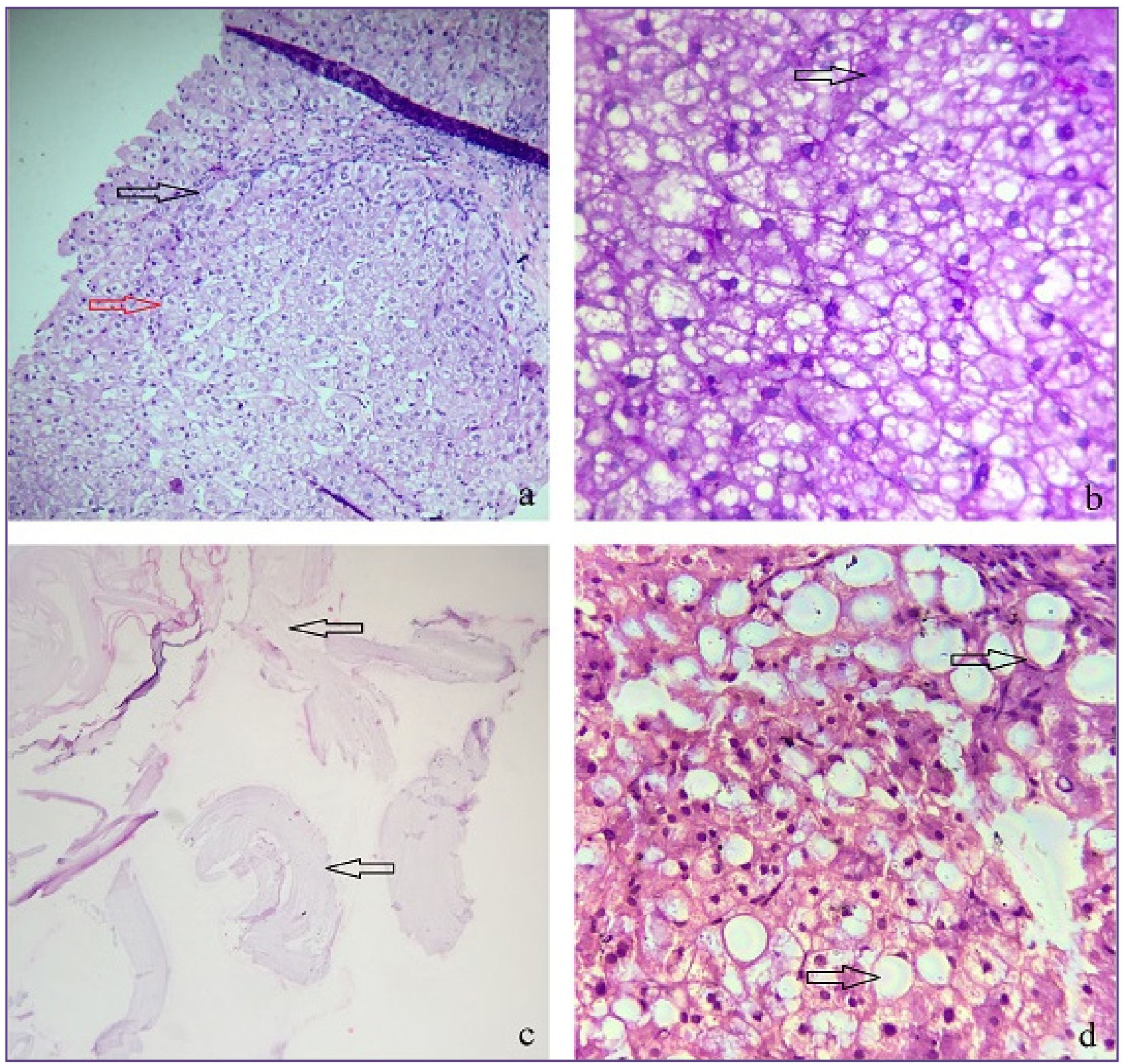

Fig. 4: Photomicrograph of cirrhosis showing nodule of regenerating hepatocytes (red arrow) and a thin fibrous septa (black arrow) (a; H\&E, x40), PAS positive hepatocytes (arrow) in glycogen storage disease (b; PAS, x40), liver hydatidosis showing laminated fibrous wall of cystic structures (arrows) (c; H\&E, x40), fatty liver disease showing macrovesicular steatosis with few inflammatory cells (arrows) (d; H\&E, x40).

\section{Discussion}

Liver being affected by various primary and secondary diseases warrants an accurate diagnosis for patient management. Therefore, liver histopathology has become a most important diagnostic tool. ${ }^{[4]}$

The spectrum of hepatic lesions varied from males to females as well as from infants to adults. In our study, the highest incidence was seen in fifth decade of life with female preponderance. This was probably due to increased alcohol intake, viral hepatic infections, obesity and other conditions during third to fifth decade of life. ${ }^{[5,6]}$ In the present study, hepatic secondaries $40 \%(n=26)$ were the commonest histopathological finding and were commonly seen among adult females. This was discrepant with the findings of Chawla et al, who reviewed and found hepatocellular carcinoma as the most common hepatic lesion with male predominance. Metastatic involvement of liver is more common than the primary hepatic tumours. ${ }^{[3]}$ 
Hepatic metastasis can be seen either by direct spread or due to the dual nature of blood supply of liver from portal and systemic circulation. The common sites of primary tumours that frequently metastasizes to liver include lung, breast, gall bladder, stomach, pancreas, and large intestine. [7] In our study, metastatic adenocarcinoma was the most frequent observation with primary tumours in gall bladder, stomach, pancreas, large bowel and breast [Figure 3a]. One case of metastatic squamous cell carcinoma was noted with primary being in oesophagus [Figure 3b]. Hepatocellular carcinoma was the most common primary hepatic malignancy encountered in our study seen in $12.3 \%(n=8)$ cases [Figure 3c]. Most of the patients were elderly females probably due to hepatitis B virus infection. One case of sarcomatoid cell hepatocellular carcinoma was seen in a 30 year old female. The second most common hepatic pathology was hepatitis $16.9 \%(n=11)$ with equal distribution among males and females during their fourth and fifth decade of life. This can be attributed to increased alcohol intake and hepatotropic viral infection. Most of the cases were of chronic hepatitis. Few cases showed concurrent cirrhosis. It was characterized by inflammatory infiltrate confined to portal tracts with intact margins. [8] Similar results were observed in the study conducted by Kringsholm B et al and Passarino G et al. ${ }^{[9,10]}$ Two cases of neonatal hepatitis were observed in 3 month old infants [Figure 3d]. They presented with jaundice, hepatomegaly and abnormal LFT. Liver biopsy showed multinucleated giant hepatocytes with severe bile stasis. Cirrhosis was the third most common hepatic lesion seen in $12.3 \%(n=8)$ cases [Figure 4a]. Gall et al has found the incidence of cirrhosis to be $6 \% .^{[11]}$ The incidence was common among females. This is most probably due to increased alcohol intake among females. ${ }^{[12]}$ Majority of cases showed interface hepatitis. Various other causes included hepatitis B infection, Wilson's disease and malnutrition. ${ }^{[5]}$ Two cases with secondary biliary cirrhosis were observed.In present study, four cases of extrahepatic biliary atresia were observed in infants who presented with persistent jaundice. Liver biopsy showed cholestasis with periportal fibrosis and inflammatory destruction of bile ducts. One case of glycogen storage disease was observed in a 20 year old female, who presented with anaemia and hepatosplenomegaly [Figure 4b]. Liver biopsy revealed glycogen deposits in hepatocytes on PAS staining. A 32 year old male patient was diagnosed with liver hydatidosis which showed cystic structures with laminating fibrous wall [Figure 4c]. Hydatid disease is a parasitic infestation caused by Echinococcus granulosus and liver is the commonest infected organ in the body. The cysts are usually localized in liver and are filled with clear fluid. ${ }^{[7]}$ One case of fatty liver (steatosis) was observed in a 48 year old female [Figure 4d]. There was no history of alcohol intake as well as no serological evidence of any viral infection. NAFLD includes a wide spectrum of pathological lesions ranging from simple steatosis to even hepatocellular carcinoma. ${ }^{[13]}$ It is frequently seen in association with various conditions like obesity, diabetes mellitus, secondary to bowel resection or bypass surgeries and consumption of steroids. Nonalcoholic steatohepatitis (NASH) is a subgroup of NAFLD. It mainly relies on liver biopsy for diagnosis. ${ }^{[14]}$

\section{Conclusion}

Histopathological examination of liver biopsy helps to diagnose and assess the severity of various hepatic diseases and often drives therapeutic management. Thus, liver biopsy is an essential tool for accurate diagnosis and management of liver diseases.

\section{Reference}

1. Pudale S.S., Ashok B.S., Ambadas P.G., Gajanan D.R., Pandharinath C.N. Study of liver pathogy in autopsy casesOriginal Article. Int J Curr Res. 2014;6(3):5795-97.

2. Theise N.D. Liver and Gall Bladder. In: Kumar V., Abbas A.K., Aster J.C. Robbins and Cotran Pathologic Basis of Disease.9th ed. South Asia: Elsevier. 2015:821-80.

3. Chawla N., Sunila S. Spectrum of histopathological findings in liver biopsy. Indian Med Gaz. 2013:363-67.

4. Parekh P.J., Majithia R., Diehl D.L., Baron T.H. Endoscopic ultrasound-guided liver biopsy. Endosc Ultrasound. 2015;4(2):85-91.

5. Uchida T., Kao H., Quispe-Sjogren M., Peters R.L. Alcoholic foamy degeneration: A pattern of acute alcoholic injury of the liver. Gastroenterology. 1983;84:683-92.

6. Ohnishi K., Iida S., Iwana S., Goto N., Nomur F., Takashi A., et al. The effect of chronic habitual alcohol intake in the development of liver cirrhosis and hepatocellular carcinoma in relation to hepatitis B surface antigen carriage. Cancer. 1982;48:672-77.

7. Desmet V.J., Rosai J. Liver. In: Rosai and Ackerman's surgical pathology.10th ed. Milan: Elsevier. 2012:857-965.

8. Umesh B.R., Gayathri B.N., Harendra Kumar M.L. Spectrum of liver pathology at autopsy. Int J Res Rev. 2015;2(3):7986.

9. Kringsholm B., Christoffersen P. Liver pathology in fatal drug addiction. Forensic Sci Int. 1982;20(2):141-51.

10. Passarino G., Ciccone G., Siragusa R., Tappero P., Mollo F. Histopathological findings in 851 autopsies, with toxicological and virological correlations. Am J Forensic Med Pathol. 2005;26(2):106-16. 
11. Gall E.A. Posthepatic, post necrotic and nutritional cirrhosis: A Pathologic analysis. Am J Pathol. 1960;36:241-70.

12. MacSween R.N.M., Scott A.R. Hepatic cirrhosis: A Clinicopathological review of 520 cases. J Clin Pathol. 1973;26:936-42.
13. Zafrani E.S. Nonalcoholic fatty liver disease. An emerging pathological spectrum.Virchows Arch. 2004;444:3.

14. Bedossa P., Poitou C., Veyrie N., Bouillot J., Basdevant A., Paradis V., Tordjman J., Clement K. Histopathological algorithm and scoring system for evaluation of liver lesions in morbidly obese patients. Hepatology. 2012;56(5):1751-59.

\section{*Corresponding author:}

Dr. M. Banyameen Iqbal, Department of Pathology, Dr. D.Y.Patil Medical College, Hospital and Research Centre Sant Tukaram Nagar,Pimpri, Pune-411018 India.

Phone: +91 9730388872

Email: banzey@gmail.com

Financial or other Competing Interests: None. 